\title{
EVALUATION OF VITEK 2 SYSTEM AND CHROMAGAR FOR IDENTIFICATION OF YEASTS IN CLINICAL LABORATORY
}

\author{
Jinal Patel ${ }^{1}$, Chirag Modi², Suman Singh ${ }^{3}$
}

${ }_{1}^{1}$ M.Sc. MLT, Department of Microbiology, Pramukhswami Medical College, Karamsad, Gujarat.

${ }_{2}^{2}$ Assistant Professor, Department of Microbiology, Pramukhswami Medical College, Karamsad, Gujarat.

3Professor and HOD, Department of Microbiology, Pramukhswami Medical College, Karamsad, Gujarat.

ABSTRACT
BACKGROUND
Successfully identifying the species of Candida is important in the treatment and management of disease as resistance acquired
by some species of Candida may avoid prescription of antifungal drugs that may not be available to species.

\section{AIM}

We evaluated the performance of VITEK 2 system and CHROMagar in identification of Candida species against the conventional culture method.

\section{SETTINGS AND DESIGN}

The study was conducted in a tertiary care hospital of Gujarat.

\section{METHODS AND MATERIAL}

The study was conducted over a period of 14 months. Candida species isolated from various clinical specimen were identified by conventional method as well as by VITEK 2 system and CHROMagar. The sensitivity and specificity of VITEK 2 system and CHROMagar was determined keeping conventional method as a gold standard method.

\section{RESULTS}

A total of 48 Candida species were identified by conventional method. Candida tropicalis $(n=26)$ and Candida albicans ( $\mathrm{n}=10)$ were most common isolates. VITEK 2 system was able to identify $36(75 \%)$ isolates whereas CHROMagar was able to identify 45 (93.75\%) isolates of Candida to species level. The sensitivity and specificity of CHROMagar for most common Candida species i.e. Candida albicans was $100 \%$ and $97.3 \%$ respectively whereas for Candida tropicalis was $100 \%$ and $95 \%$ respectively. The sensitivity and specificity of VITEK 2 system for Candida albicans was $100 \%$ whereas for Candida tropicalis was $76.92 \%$ and $100 \%$ respectively.

\section{CONCLUSIONS}

We concluded that CHROMagar could be routinely used for identification of commonly isolated Candida species whereas VITEK 2 system could be used for determining MICs of antifungal agents.

\section{KEYWORDS}

Candida, VITEK 2 System, CHROMagar, Sensitivity, Specificity.

HOW TO CITE THIS ARTICLE: Patel J, Modi C, Singh S. Evaluation of Vitek 2 system and CHROMagar for identification of yeasts in clinical laboratory. J. Evolution Med. Dent. Sci. 2016;5(60):4204-4206, DOI: 10.14260/jemds/2016/959

\begin{abstract}
INTRODUCTION
Candida species among various yeasts are common human commensals that cause a wide variety of diseases both in immunocompromised and immunocompetent individuals. ${ }^{1,2}$ Identification of yeast by conventional method is labour intensive and time consuming. ${ }^{3}$ To overcome this, chromogenic media (CHROMagar) and commercial automated identification systems like VITEK 2 are available. ${ }^{3,4}$ Each of these methods has their own advantages. Comparative studies have given variable results. This study aims to evaluate the performance of a commercially available CHROMagar and VITEK 2 automated system for the identification of clinically important yeasts against the conventional culture method.
\end{abstract}

Financial or Other, Competing Interest: None.

Submission 13-06-2016, Peer Review 14-07-2016,

Acceptance 20-07-2016, Published 28-07-2016.

Corresponding Author:

Dr. Chirag Modi,

B/117, Shrenik Park,

Opp. Akota Stadium,

Productivity Road,

Akota, Vadodara-390020,

Gujarat.

E-mail: chiragmm@charutarhealth.org

DOI: $10.14260 /$ jemds/2016/959

\section{SUBJECTS AND METHODS}

This prospective comparative study was conducted from June 2013 to September 2014 in microbiology laboratory of a tertiary care hospital in rural part of Gujarat after approval by institutional ethics committee. Consecutive strains of Candida isolated from various clinical specimens were included. The comparison was made between conventional method, VITEK 2 automated system, and CHROMagar in terms of its species identification of Candida isolates. Conventional method was considered to be the gold standard.

Conventional isolation and identification of yeast was performed by inoculating clinical specimen on Sabouraud Dextrose Agar (SDA). The plates were examined after 24 hours for the growth of colonies. These colonies were further followed by performing germ tube test, morphology on corn meal agar, sugar fermentation, and assimilation test for identification of Candida species through conventional method. The average time taken for identification of Candida species by conventional method was 72-96 hours. The colonies from 24 hours growth on SDA were used for VITEK 2 automated system. Species identification was provided by VITEK 2 automated system after 24 hours of incubation. The average time taken for identification of Candida species by 
VITEK 2 automated system was 48 hours. Simultaneously, the specimen was inoculated on CHROMagar and species were identified after 24 hours based on their characteristics colony colours as per the manufactures instructions. ${ }^{5}$

The sensitivity and specificity of CHROMagar and VITEK 2 automated system was calculated in comparison to conventional method.

\section{RESULTS}

Out of 50 isolates of Candida species that were isolated from the clinical specimens, 48 were identified to species level by conventional methods and were included further in the study. Of these, C. tropicalis was the most common i.e. $54.16 \%(n=26)$ followed by C. albicans $20.8 \%(n=10)$, C. glabrata $10.41 \%$ $(\mathrm{n}=5)$, C. parapsilosis $12.5 \%(\mathrm{n}=6)$, and C. krusei $2.08 \%(\mathrm{n}=1)$ All 10 isolates of $\mathrm{C}$. albicans were positive for germ tube test whereas the remaining 40 isolates including the two isolates that were not identified by conventional method were negative for germ tube test. When compared with conventional method, VITEK 2 system was able to identify 36 (75\%) isolates whereas CHROMagar was able to identify 45 (93.75\%) isolates. Comparison of various species isolated by all three methods is shown in figure 1 and sensitivity and specificity for each species by these methods has been calculated as shown in Table 1.

\begin{tabular}{|c|c|c|c|c|}
\hline \multirow{2}{*}{$\begin{array}{c}\text { Candida } \\
\text { Species }\end{array}$} & \multicolumn{2}{|c|}{ CHROMagar } & \multicolumn{2}{c|}{ VITEK 2 system } \\
\cline { 2 - 5 } & $\begin{array}{c}\text { Sensiti- } \\
\text { vity (\%) }\end{array}$ & $\begin{array}{c}\text { Specifi- } \\
\text { city (\%) }\end{array}$ & $\begin{array}{c}\text { Sensiti- } \\
\text { vity (\%) }\end{array}$ & $\begin{array}{c}\text { Specifi- } \\
\text { city (\%) }\end{array}$ \\
\hline $\begin{array}{c}\text { Candida } \\
\text { albicans }\end{array}$ & 100 & 97.3 & 100 & 100 \\
\hline $\begin{array}{c}\text { Candida } \\
\text { tropicalis }\end{array}$ & 100 & 95 & 76.9 & 100 \\
\hline $\begin{array}{c}\text { Candida } \\
\text { parapsilosis }\end{array}$ & 66.6 & 100 & 66.6 & 100 \\
\hline $\begin{array}{c}\text { Candida } \\
\text { glabrata }\end{array}$ & 80 & 100 & 40 & 100 \\
\hline $\begin{array}{c}\text { Candida } \\
\text { krusei }\end{array}$ & 100 & 93.8 & 100 & 100 \\
\hline \multicolumn{4}{|c|}{$\begin{array}{c}\text { Table 1: Sensitivity and Specificity of Different } \\
\text { Methods in Identifying Candida Species (n=48) }\end{array}$} \\
\hline
\end{tabular}

\section{DISCUSSION}

Invasive candidiasis is one of the major causes of morbidity and mortality in both immunocompromised and immunecompetent patients, the crude and attributable mortality being as high as $20 \%$ and $40 \%$ respectively. 6,7

Accurate species identification of Candida is important for timely and effective treatment of the infections as the nonalbicans species continue to be increasingly isolated and their response to the antifungal therapy is highly variable. Most of the fluconazole-resistant species recovered from clinical specimens particularly blood have been non-albicans. ${ }^{8}$

In present study, Candida non-albicans formed major group of isolates $(80.2 \%)$ where $C$. tropicalis accounted for $54.1 \%(n=26)$ of all isolates. Jain et al. also reported Candida non-albicans as major group of all isolates (86\%) with $\mathrm{C}$. tropicalis accounting for $64 \%$ of all isolates. The reason for emergence of non-albicans species as human pathogen could be attributed to a higher amount of resistance to antifungal agents in non-albicans spp. when compared to C. albicans thus giving a selective advantage to these species. ${ }^{9}$
In our study, 48 Candida species were identified by conventional method. VITEK 2 system was able to identify 34 (Sensitivity $=71 \%$ ) of these isolates, nine isolates $(19 \%)$ were falsely identified and five isolates $(10 \%)$ were not identified. In contrast, 45 isolates (sensitivity $=94 \%$ ) were accurately identified by CHROMagar and three isolates (6\%) were incorrectly identified.

As seen in the Table 1, sensitivity and specificity of VITEK 2 with respect to conventional method for C. krusei and C. albicans was $100 \%$. CHROMagar was found to give $100 \%$ sensitivity for C. albicans, C. tropicalis, and C. krusei with a specificity of $97.3 \%, 95 \%$, and $93.87 \%$ respectively. VITEK 2 gave lowest sensitivity in identification of C. glabrata i.e. $40 \%$, but with $100 \%$ specificity. C. parapsilosis was identified with low sensitivity of $66.66 \%$ by both VITEK 2 and CHROMagar with $100 \%$ specificity.

Thus, we found CHROMagar to be more sensitive in comparison to VITEK 2 i.e. $94 \%$ vs $71 \%$. This is in contrast to the finding by Agarwal et al who found VITEK 2 system more sensitive $(97 \%$ vs $86 \%)$ in comparison to CHROMagar. Sensitivity of identification by CHROMagar in their study was $100 \%$ for C. rugosa followed by $89 \%, 80 \%$, and $77 \%$ for C. albicans, C. tropicalis, and C. parapsilosis respectively. ${ }^{10}$ Baradkar et al found CHROMagar sensitivity and specificity of $60-100 \%$ and $88-100 \%$ respectively for various Candida species, which is similar to our findings. They also reported $100 \%$ sensitivity and specificity in identification of C. tropicalis. For other species, it was C. albicans (96.55 and 96.42\%); C. parapsilosis (80 and 98.03\%); C. glabrata (90.90 and 88.23\%); and C. dubliniensis (60 and 96.55\%) respectively. ${ }^{11}$

In a study by Sood et al, VITEK system and conventional method were found to have an agreement of $68 \%$ with respect to identification of Candida species. Moreover, VITEK 2 system identified 14 Candida species where conventional method had failed. ${ }^{12}$ In a study by Jain et al, VITEK 2 had lower sensitivity compared to CHROMagar with agreement of $49 \%$, 39\%, and $31 \%$ for C. tropicalis, C. albicans, and C. parapsilosis respectively, but this was $100 \%$ for C. rugosa. ${ }^{13}$ We had a low sensitivity of $40 \%$ for C. glabrata by VITEK.

Thus, CHROMagar and VITEK 2 system have given variable sensitivity and specificity. CHROMagar was able to identify majority of common isolates i.e. C. albicans, C. tropicalis, C. glabrata, and C. krusei in the present study. VITEK 2 system could identify two out of three Candida species where CHROMagar failed. Hence, CHROMagar supported by VITEK 2 system could be effectively used in identification of Candida.

Identification of Candida species by conventional method may take up to 72 hours or more, which is possible within 2448 hours by CHROMagar with a high sensitivity and is recommended as a method of identification from clinical specimens. VITEK 2 system in contrast has a lower sensitivity compared to CHROMagar, but has an advantage of giving minimum inhibitory concentrations of antifungal agents, which currently is required for management of patients as drug resistance is becoming increasingly common amongst various Candida isolates. Moreover, VITEK 2 system is an automated system requiring an electrical backup and other accessories for processing of an isolate. The system utilizes special cards for identification of Candida species and for determining their antimicrobial susceptibility patterns. Apart from this, it requires a proper quality control procedure to 
ensure that the results provided by the system are accurate thus making VITEK 2 system a costly affair. However, a major advantage of using this system is its ability to provide minimum inhibitory concentrations of various antifungal agents. On the other hand, CHROMagar is a cheaper alternative for identification of Candida species and does not require extensive technology and skill to identify the isolate. However, CHROMagar could only be used for rapid identification of isolate and does not provide a benefit of determining antifungal susceptibility as with VITEK 2 system. An early identification of Candida species done by CHROMagar, however, could be of great help in clinical decision making and patient's outcome.

Based on the findings of the present study and pros and cons of both the identification systems, it was concluded that CHROMagar could be routinely used as a medium for rapid identification of Candida species whereas VITEK 2 system could be used in support to CHROMagar and for determining minimum inhibitory concentrations of antifungal agents. A limitation of the study was that the number of isolates used for comparison of the two methods was fairly low owing to a smaller number of clinical specimens being received for fungal culture. However, the authors would recommend performing a similar kind of study with large number of isolates to validate the results of the present study.

\section{REFERENCES}

1. Madhavan P, Jamal F, Chong PP. Laboratory isolation and identification of candida species. J Appl Sciences 2011;11:2870-7.

2. Graf B, Adam T, Zill E, et al. Evaluation of VITEK 2 system for rapid identification of yeasts and yeast-like organisms. J Clin Microbiol 2000;38(5):1782-5.

3. Aubertine CL, Rivera M, Rohan SM, et al. Comparative study of the new colorimetric VITEK 2 yeast identification card versus the older fluorometric card and of CHROMagar candida as a source medium with the new card. J Clin Microbiol 2006;44(1):227-8.
4. Wadlin JK, Hanko G, Stewart R, et al. Comparison of three commercial system for identification of yeasts commonly isolated in the clinical microbiology laboratory. J Clin Microbiol 1999;37(6):1967-70.

5. Hospenthal DR, Beckius ML, Floyd KL, et al. Presumptive identification of candida species other than C. albicans, C. krusei, and C. tropicalis with the chromogenic medium CHROMagar candida. Ann Clin Microbiol Antimicrob 2006;5:1.

6. Lu JJ, Lee SY, Chiueh TS. In vitro antifungal susceptibility testing of candida blood isolates and evaluation of the Etest method. J Microbiol Immunol Infect 2004;37(6):33542.

7. Méan M, Marchetti O, Calandra T. Bench-to-bedside review: candida infections in the intensive care unit. Crit Care 2008;12(1):204.

8. Dharwad S, Dominic SRM. Species identification of candida isolates in various clinical specimens with their antifungal susceptibility patterns. J Clin and Diag Res 2011;5(6):1177-81.

9. Sobel JD. The emergency of non-albicans candida species as causes of invasive candidiasis and candidaemia. Curr Infect Dis Rep 2006;8(6):427-33.

10. Agarwal S, Manchanda V, Verma $\mathrm{N}$, et al. Yeast identification in routine clinical microbiology laboratory and its clinical relevance. Ind J Med Microbiol 2011;29(2): 172-7.

11. Baradkar VP, Mathur M, Kumar S. Hichrom candida agar for identification of candida species. Ind J Patho Microbiol 2010;53(1):93-5.

12. Sood P, Mishra B, Dogra V, et al. Comparison of VITEK yeast biochemical card with conventional method for speciation of candida. Ind J Pathol Microbiol 2000;43(2): 143-5.

13. Jain N, Mathur P, Misra MC, et al. Rapid identification of yeast isolates from clinical specimens in critically ill ICU patients. J Lab Physicians 2012;4(1):30-4. 